\title{
3 SÍNDROME DE BURNOUT E ESTILO DE VIDA EM ESTUDANTES DE ENSINO MÉDIO
}

\author{
| Anna Santos¹; Henrique Mineiro²; Lucas Cruz ${ }^{3}$; Luiz Santos ${ }^{4}$; Marise Silveira ${ }^{5}$; Lucineia de Pinho ${ }^{6} \mid$
}

\section{RESUMO}

INTRODUÇÃO: Os estudantes do ensino médio vivenciam nesta fase experiências estressantes, como a escolha de qual profissão seguir e os preparativos para os exames admissionais, o que pode predispor ao desenvolvimento da Síndrome de Burnout.

OBJETIVO: Este estudo teve como objetivo analisar a relação entre a Síndrome de Burnout e o estilo de vida dos estudantes de ensino médio. MÉTODOS: Estudo transversal, quantitativo, realizado com estudantes do ensino médio. Para a coleta de dados foram utilizados o questionário "Estilo de Vida Fantástico" e o "Maslach Burnout Inventory - Student Survey (MBI-SS)". Para estimar e testar a associação entre as dimensões da Síndrome de Burnout e estilo de vida entre os estudantes empregou-se o coeficiente de correlação de Pearson.

RESULTADOS: Participaram do estudo 966 estudantes, com média de idade de 16,61 anos. Observou-se que 12,1\% apresentaram indicativos de Síndrome de Burnout. Observou-se relação negativa entre exaustão emocional e sono, cinto de segurança, estresse e sexo seguro $(-0,225 ; \mathrm{p}<0,001)$; descrença e cigarro e drogas $(-0,206 ; \mathrm{p}<0,001)$; e positiva para eficácia profissional, família e amigos $(0,224 ; \mathrm{p}<0,001)$, sono, cinto de segurança, estresse e sexo seguro $(0,282 ; \mathrm{p}<0,001)$ e introspecção $(0,245 ; \mathrm{p}<0,001)$.

CONCLUSÕES: Pode-se concluir que práticas saudáveis de vida adotadas pelos estudantes de ensino médio podem ser relacionadas a baixos índices de estresse nesta fase.

\section{DESCRIPTORES: Esgotamento profissional; Estudantes; Saúde pública}

\section{RESUMEN}

\section{"Síndrome de burnout y estilo de vida en estudiantes de ense-} nanza media"

CONTEXTO: Los estudiantes de la enseñanza media experimentan en esta fase experiencias estresantes, como la elección de qué profesión seguir y los preparativos para los exámenes de admisión, lo que puede predisponer al desarrollo del Síndrome de Burnout.

OBJETIVO: Este estudio tuvo como objetivo verificar la correlación entre el Síndrome de Burnout y el estilo de vida de los estudiantes de enseñanza media.

MÉTODOS: Estudio transversal, cuantitativo, realizado con estudiantes de la enseñanza media. Para recoger los datos, se utilizaron el cuestionario "Estilo de Vida Fantástico" y el "Maslach Burnout Inventory". Para estimar y probar la asociación entre las dimensiones de la Síndrome de Burnout y el estilo de vida entre los estudiantes, se empleó el coeficiente de correlación de Pearson.

RESULTADOS: El estudio tuvo la participación de 966 estudiantes, con un promedio de edad de 16,61 años. Se observó que el 12,1\% presentaba indicativos de Síndrome de Burnout. Se observó una relación negativa entre agotamiento emocional y sueño, cinturón de seguridad, estrés y sexo seguro $(-0,225 ; \mathrm{p}<0,001)$; incredulidad y el cigarrillo y las drogas $(-0,206$, $\mathrm{p}<0,001)$; y positiva para eficacia profesional, familia y amigos $(0,224$; $\mathrm{p}<0,001)$, sueño, cinturón de seguridad, estrés y sexo seguro $(0,282 ; \mathrm{p}$ $<0,001)$ e introspección $(0,245 ; \mathrm{p}<0,001)$.

CONCLUSIONES: Se puede concluir que las prácticas saludables de vida adoptadas por los estudiantes de la enseñanza media pueden estar relacionadas con los bajos índices de estrés en esta fase.

\section{PALAVRAS-CHAVE: Agotamiento profesional; Estudiantes; Salud pública}

\section{ABSTRACT \\ "Burnout syndrome and lifestyle in high school students"}

BACKGROUND: High school students deal with stressful experiences at this stage, such as the choice of which profession to follow and the training for admission exams, which may predispose to the development of Burnout Syndrome.

AIM: This study aimed to verify the correlation between Burnout Syndrome and the lifestyle of high school students.

METHODS: This is a cross-sectional and quantitative study performed with high school students. In order to collect data, we used the questionnaire "Fantastic Lifestyle" and "Maslach Burnout Inventory". In order to estimate and test the association between the dimensions of Burnout Syndrome and the lifestyle among students, we used the Pearson's correlation coefficient.

RESULTS: This study was attended by 966 students, with a mean age of 16.61 years. We observed that $12.1 \%$ presented signs of Burnout Syndrome. We observed a negative relationship among emotional exhaustion and sleep, seatbelt, stress and safe sex $(-0.225 ; \mathrm{p}<0.001)$; disbelief and cigarettes and drugs $(-0.206 ; \mathrm{p}<0.001)$; and positive for professional effectiveness, family and friends $(0.224 ; \mathrm{p}<0.001)$, sleep, seatbelt, stress and safe $\operatorname{sex}(0.282 ; \mathrm{p}<0.001)$ and introspection $(0.245 ; \mathrm{p}<0.001)$.

CONCLUSIONS: We can be conclude that healthy life practices adopted by high school students can be related to low stress indexes at this stage.

\section{KEYWORDS: Burnout, professional; Students; Public health}

Submetido em 30-09-2018

Aceite em $04-12-2018$

\footnotetext{
1 Académica em Medicina pelas Faculdades Integradas Pitágoras de Montes Claros, 39408-007 Montes Claros (MG), Brasil, karolzinha_rfs@hotmail.com 2 Académico em Medicina pelas Faculdades Integradas Pitágoras de Montes Claros, 39408-007 Montes Claros (MG), Brasil, henriquegmineiro@gmail.com 3 Académico em Medicina pelas Faculdades Integradas Pitágoras de Montes Claros, 39408-007 Montes Claros (MG), Brasil, lucasperth2014@gmail.com 4 Académico em Medicina pelas Faculdades Integradas Pitágoras de Montes Claros, 39408-007 Montes Claros (MG), Brasil, luizsantoz1@yahoo.com.br 5 Doutora em Ciências da Saúde; Docente na Universidade Estadual de Montes Claros, 39401-089 Montes Claros (MG), Brasil, ciaestatistica@yahoo.com.br 6 Doutora em Ciências da Saúde; Docente na Universidade Estadual de Montes Claros; Docente nas Faculdades Integradas Pitágoras de Montes Claros, $39408-007$ Montes Claros (MG), Brasil, lucineiapinho@hotmail.com
}

Citação: Santos, A. K., Mineiro, H., Cruz, L., Santos, L., Silveira, M., \& Pinho, P. (2019). Síndrome de burnout e estilo de vida em estudantes de ensino médio. Revista Portuguesa de Enfermagem de Saúde Mental (21), 16-xx. 


\section{INTRODUÇÃO}

A sociedade atual, caracterizada pelo progresso tecnológico, exige sucesso imediato, padronização de perfis intelectuais, culturais e corporais, o que gera uma alta demanda para o indivíduo. Na tentativa de se adaptar a esses padrões, há a possiblidade de um desequilíbrio ao considerar a capacidade individual e as exigências do ambiente, denominado estresse (Marques, Gasparotto, e Coelho, 2015). Devido às exigências mercadológicas e sociais, o estresse tem aumentado na vida das pessoas em diversos segmentos, incluindo o âmbito estudantil (Tuominen-Soini, \& Salmela-Aro, 2014; Marques et al., 2015).

O ensino médio constitui a última etapa da educação básica, sendo pré-requisito para o ingresso no ensino superior.

Nessa etapa, os estudantes precisam decidir sobre qual profissão seguir e se prepararem para a realização dos exames admissionais.

Esse preparo é considerado uma situação estressora ambiental duradoura, podendo gerar importantes efeitos psicopatológicos nos educandos. O momento de escolha profissional constitui período muito conflituoso para os jovens do ensino médio, já que além das próprias dificuldades da idade, bem como as mudanças físicas e psicológicas que experienciam, estes ainda devem considerar as implicações que suas escolhas trarão ao seu futuro (Faria, Weber, e Ton, 2012; Gonzaga, Silva, e Enumo, 2016).

Por vivenciarem uma fase de transição, os estudantes de ensino médio estão sujeitos às novas experiências e, ao mesmo tempo, são compelidos a renunciar sua própria vida social em prol do acesso à instituição de ensino superior, assim, muitas vezes como efeito colateral emerge o estresse exaustivo - denominado como Síndrome de Burnout (SB) (Tuominen-Soini, \& Salmela-Aro, 2014). Essa exaustão configura a primeira dimensão da síndrome, associando-se ao absenteísmo estudantil, fadiga, declínio das capacidades de memória e concentração (Galdino, Martins, Haddad, Robazzi, e Birolim, 2016).

Surgem ainda outros sintomas, como nervosismo, sentimentos de desamparo, vazio, solidão e tristeza que, em longo prazo, tornam-se gatilhos para o desenvolvimento de outras patologias relacionadas ao humor deprimido e ansiedade (Ovchinnikov, Palchenkova, \& Kalachev, 2015; Galván-Molina, Jiménez-Capdeville, Hernández-Mata, \& Arellano-Cano, 2017).
Essas manifestações repercutem de forma negativa na estrutura familiar e pessoal do indivíduo, por conseguinte, refletindo na qualidade e desempenho das atividades realizadas (Tomaschewski-Barlem et al., 2014; Magri, Melchior, Jarina, Simonaggio, e Bataglion, 2016); Galván-Molina et al., 2017).

Os indivíduos com SB, por vezes, também manifestam sintomas físicos, tais como cefaleia e insônia, seguidos de problemas musculoesqueléticos (Magri et al., 2016). Há, ainda, manifestação de problemas imunológicos, como aumento da frequência de reações alérgicas (Ovchinnikov et al., 2015).

Nesse sentido, o objetivo da pesquisa foi verificar a correlação entre a Síndrome de Burnout e o estilo de vida dos estudantes de ensino médio.

\section{MÉTODOS}

O presente trabalho faz parte da pesquisa "Estudantes on line: uso e dependência da Internet”. A população foi constituída de estudantes de ensino médio, regularmente matriculados em escolas públicas e particulares, da $1^{\text {a }}$ a $3^{\text {a }}$ série da cidade de Montes Claros, Minas Gerais. O tamanho amostral foi definido considerando a prevalência do evento de $50 \%$, nível de confiança de $95 \%$, erro padrão de $5 \%$. Foi adotada a correção para o efeito de delineamento (Deff=2,0) e estabeleceu-se também um acréscimo de $15 \%$ para compensar as possíveis não respostas e perdas. Estimou-se a participação de no mínimo 884 adolescentes. Utilizou-se como critério de inclusão ser estudante regularmente matriculado na instituição e na turma selecionada. Como critério de exclusão, considerou-se o fato de o estudante não estar presente no dia agendado para a coleta de dados ou não entregar o Termo de Assentimento e/ou Consentimento Livre e Esclarecido.

Como instrumento de coleta de dados, utilizou-se um questionário que contemplava as variáveis sociodemográficas (sexo, idade e classe econômica) e de formação (instituição de estudo, turno e série). Foram utilizados o questionário "Estilo de Vida Fantástico" e o "Maslach Burnout Inventory - Student Survey (MBI-SS)”.

Para avaliação do estilo de vida dos adolescentes, aplicou-se o questionário validado "Estilo de Vida Fantástico" (Rodriguez Añez, Reis, e Petroski, 2008). Este questionário considera o comportamento dos estudantes no último mês. $\mathrm{O}$ instrumento compreende 25 questões distribuídas em nove domínios (23 em escala Likert de zero a quatro pontos e duas questões dicotômicas não; zero e sim; quatro pontos). 
Foram considerados os domínios: família e amigos (2 itens); atividade física ( 2 itens); nutrição (3 itens); cigarro e drogas (4 itens); álcool (3 itens); sono, cinto de segurança, estresse e sexo seguro (5 itens); tipo de comportamento (2 itens); introspecção (3 itens) e trabalho (1 item). A soma de todos os pontos permite chegar a um escore total que classifica os indivíduos em cinco categorias que são: "Excelente" (85 a 100 pontos); "Muito bom" (70 a 84 pontos); "Bom" (55 a 69 pontos); "Regular" (35 a 54 pontos); e "Necessita melhorar" (0 a 34 pontos). Para cada um dos domínios também foi realizada a classificação em estilo de vida saudável e estilo de vida não saudável. Tal classificação foi realizada conforme a possibilidade de pontos possíveis em cada um dos domínios. Para o presente estudo, a categoria Necessita melhorar é a relatada como a que apresenta um estilo de vida não saudável ou inadequado.

A fim de avaliar os sintomas da Síndrome de Burnout, utilizou-se o "Maslach Burnout Inventory - Student Survey (MBI-SS)" (Maroco, e Tecedeiro, 2009). O inventário é constituído por 15 itens subdivididos em três dimensões: exaustão emocional, descrença e eficácia profissional. Cada item deve ser avaliado em uma pontuação que vai de 0 a 6 , sendo 0 nunca, 1 uma vez por ano ou menos, 2 uma vez por mês ou menos, 3 algumas vezes por mês, 4 uma vez por semana, 5 algumas vezes por semana e 6 todos os dias. De acordo com o MBI-SS, a dimensão exaustão emocional pode ser classificado em baixo (0-9), moderado (10-14) e alto (>14); a dimensão descrença classificado em baixo (0-1), moderado (2-6) e alto ( $>6)$; a dimensão eficácia profissional classificado em baixo $(\leq 22)$, moderado $(23-27)$ e alto $(\geq 28)$.O indivíduo é considerado portador de Síndrome de Burnout quando sua pontuação atinge escore alto para exaustão (escore maior que 14), escore alto para descrença (escore maior do que 6) e escore baixo para eficácia profissional (escore menor que 23).

A coleta dos dados foi realizada em sala de aula, entre o segundo semestre de 2016 e o primeiro semestre de 2017.

O instrumento foi entregue, após autorização dos professores, sendo respondido pelos estudantes e recolhido, logo após, juntamente com o Termo de Consentimento Livre e Esclarecido (TCLE) assinado pelos pais dos estudantes, juntamente com o termo de assentimento assinado pelos mesmos.

Para análise dos dados, utilizou-se o software estatístico SPSS (Statistical Package for Social Sciences), versão 20.0 .
Os resultados referentes à amostra estudada foram obtidos por meio de estatística descritiva (frequência simples, percentual, média e desvio padrão). Utilizou-se o coeficiente de correlação de Pearson para estimar e testar a correlação linear entre as dimensões da SB e estilo de vida entre os estudantes. A significância estatística foi estabelecida em $5 \%(\mathrm{p}<0,05)$. O coeficiente de correlação de Pearson de $r \geq 0,2$ ou $\leq-0,2$ foi considerado como associação adequada.

Este estudo, cumprindo o recomendado da Resolução $\mathrm{n}^{\circ} 466$, de 12 de dezembro de 2012, e da Norma Operacional $n^{\circ} 001 / 2013$, de 30 de setembro de 2013, do Conselho Nacional de Saúde/Ministério da Saúde, do Conselho Nacional de Saúde, foi aprovado pelo Comitê de Ética em Pesquisa sob protocolo no 1.520.173/2016.

\section{RESULTADOS}

Participaram desta pesquisa 966 estudantes do ensino médio, sendo $53 \%(n=516)$ do sexo feminino e $47 \%$ do sexo masculino $(n=450)$. A média de idade foi de 16,61 $( \pm 1,26)$ anos.

Entre os estudantes, 16,3\% foram classificados na classe econômica A, 39,4\% na classe econômica B, 38,5\% na classe econômica C e 5,8\% na classe econômica D/E. Quanto à série, observou-se que $42,9 \%$ cursavam o $1^{\circ}$ ano do ensino médio, $33,1 \%$ o segundo ano e $24,0 \%$ o terceiro ano. A maioria dos estudantes frequentava as aulas no turno matutino $(91,4 \%)$.

Com relação à classificação da Síndrome de Burnout, identificou-se que $12,1 \%(n=117)$ dos estudantes a apresentaram.

$\mathrm{Na}$ Tabela 1 são apresentadas as medidas descritivas para as dimensões exaustão emocional, descrença e eficácia profissional.

Tabela 1 - Descrição dos Valores Médios e Desvio Padrão dos Domínios da Síndrome Burnout em Estudantes do Ensino Médio

\begin{tabular}{|l|l|}
\hline Domínios & Média (DP) \\
\hline Exaustão emocional (EE) & $2,35 \pm 1,31$ \\
\hline Descrença (DE) & $1,70 \pm 1,27$ \\
\hline Eficácia profissional (EP) & $3,45 \pm 1,41$ \\
\hline
\end{tabular}

$\mathrm{Na}$ análise do estilo de vida dos estudantes, verificou-se que $102(10,6 \%)$ foram classificados com estilo de vida excelente, $432(44,7 \%)$ muito bom, $332(34,4 \%)$ bom, $61(6,3 \%)$ regular e $5(0,5 \%)$ necessita melhorar. As medidas descritivas das dimensões do Estilo de Vida Fantástico (média e desvio padrão) estão apresentadas na Tabela 2. 
Tabela 2 - Descrição dos Valores Médios e Desvio Padrão dos Domínios do Estilo de Vida em Estudantes do Ensino Médio

\begin{tabular}{|l|l|}
\hline Domínios & Média (DP) \\
\hline Família e amigos & $4,25 \pm 1,70$ \\
\hline Atividade física & $2,60 \pm 2,01$ \\
\hline Nutrição & $4,65 \pm 2,19$ \\
\hline Cigarro e drogas & $12,04 \pm 1,65$ \\
\hline Álcool & $8,35 \pm 1,67$ \\
\hline $\begin{array}{l}\text { Sono, Cinto de Segurança, } \\
\text { Estresse e Sexo Seguro }\end{array}$ & $11,20 \pm 3,40$ \\
\hline Tipo de comportamento & $3,41 \pm 1,49$ \\
\hline Introspecção & $6,33 \pm 2,25$ \\
\hline Trabalho & $3,41 \pm 1,12$ \\
\hline
\end{tabular}

$\mathrm{Na}$ Figura 1 foram apresentadas as prevalências do estilo de vida inadequado ao considerar a análise por domínios do instrumento utilizado. Entre os estudantes investigados, 46,2\% dos entrevistados apresentaram inadequação para atividade física. $\mathrm{Na}$ sequência, o domínio mais prevalente foi o Tipo de comportamento, em que $24,0 \%$ dos estudantes estavam inadequados quanto a esse hábito de vida. Ainda, 20,3\% e 18,9\% dos estudantes relataram hábito de vida inadequado para nutrição e família e amigos, respetivamente.

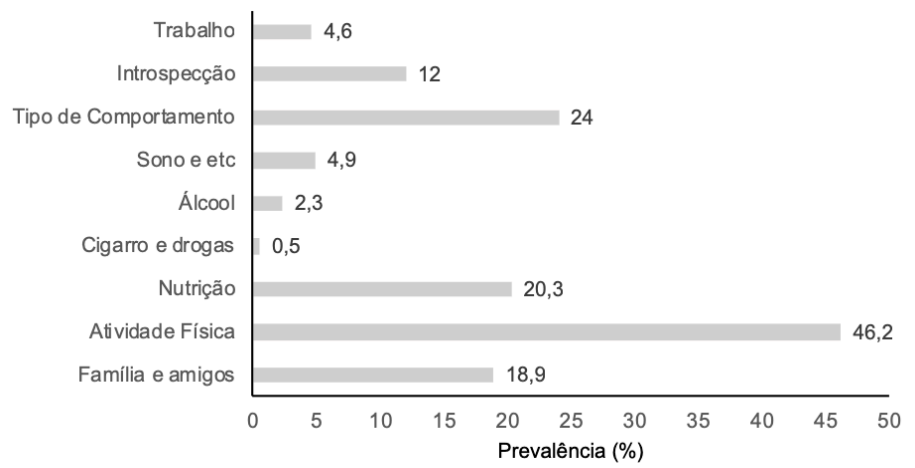

Figura 1 - Prevalência do estilo de vida inadequado para a classificação de acordo com os domínios do estilo de vida em estudantes de ensino médio.

$\mathrm{Na}$ análise de correlação entre as dimensões da SB e do estilo de vida dos estudantes, observaram-se correlações lineares significativas $(\mathrm{p}<0,05)$ para todos os itens das dimensões, exceto para as relações entre as dimensões descrença e atividade física, e entre as dimensões família e amigos e exaustão emocional (Tabela 3). A correlação foi fortemente negativa para as dimensões exaustão emocional e sono, cinto de segurança, estresse e sexo seguro $(-0,225, \mathrm{p}<0,001)$; descrença e cigarro e drogas $(-0,206, \mathrm{p}<0,001)$; e positiva para eficácia profissional, família e amigos $(0,224, \mathrm{p}<0,001)$, sono, cinto de segurança, estresse e sexo seguro $(0,282$, $\mathrm{p}<0,001)$ e introspecção $(0,245, \mathrm{p}<0,001)$.

Tabela 3 - Relação entre os Domínios da Síndrome Burnout e de Estilo de Vida dos Estudantes do Ensino Médio

\begin{tabular}{|l|l|l|l|l|l|l|}
\hline Domínios & $\begin{array}{l}\text { Exaustão } \\
\text { emocio- } \\
\text { nal }\end{array}$ & $\mathbf{p}$ & $\begin{array}{l}\text { De- } \\
\text { scrença }\end{array}$ & $\mathbf{p}$ & $\begin{array}{l}\text { Eficácia } \\
\text { profis- } \\
\text { sional }\end{array}$ & $\mathbf{p}$ \\
\hline $\begin{array}{l}\text { Família e } \\
\text { amigos }\end{array}$ & $-0,019$ & 0,560 & $-0,123$ & $<0,001$ & 0,224 & $<0,001$ \\
\hline $\begin{array}{l}\text { Atividade } \\
\text { física }\end{array}$ & $-0,064$ & 0,047 & $-0,010$ & 0,755 & 0,137 & $<0,001$ \\
\hline Nutrição & $-0,142$ & $<0,001$ & $-0,123$ & $<0,001$ & 0,180 & $<0,001$ \\
\hline $\begin{array}{l}\text { Cigarro e } \\
\text { drogas }\end{array}$ & $-0,154$ & $<0,001$ & $-0,206$ & $<0,001$ & 0,136 & $<0,001$ \\
\hline Álcool & $-0,093$ & 0,004 & $-0,132$ & $<0,001$ & 0,163 & $<0,001$ \\
\hline $\begin{array}{l}\text { Sono, } \\
\text { Cinto de } \\
\text { Segu- } \\
\text { rança, } \\
\text { Estresse } \\
\text { e Sexo } \\
\text { Seguro }\end{array}$ & $-0,225$ & $<0,001$ & $-0,190$ & $<0,001$ & 0,282 & $<0,001$ \\
\hline $\begin{array}{l}\text { Tipo de } \\
\text { comporta- } \\
\text { mento }\end{array}$ & $-0,149$ & $<0,001$ & $-0,136$ & $<0,001$ & 0,093 & 0,004 \\
\hline $\begin{array}{l}\text { Intro- } \\
\text { specção }\end{array}$ & $-0,161$ & $<0,001$ & $-0,178$ & $<0,001$ & 0,245 & $<0,001$ \\
\hline Trabalho & $-0,118$ & $<0,001$ & $-0,165$ & $<0,001$ & 0,156 & $<0,001$ \\
\hline
\end{tabular}

Coeficiente de correlação de Pearson

\section{DISCUSSÃO}

Ao analisar o perfil dos estudantes do ensino médio, pode-se observar que a prevalência da síndrome de Burnout nesses estudantes se encontrava próxima àquela apresentada em estudo de coorte realizado por Tuominen-Soini, \& Salmela-Aro (2014), no qual foi constatada a prevalência de síndrome de Burnout em $14 \%$ dos estudantes da educação finlandesa. Esses dados se aproximam de estudo com estudantes universitários no cenário nacional (Costa, Santos, Santos, Melo, \& Andrade, 2012; Magri et al., 2016; Chagas, Moreira Junior, Cunha, Caixeta, e Fonseca, 2016). Entretanto, quando analisada de forma segmentada, no âmbito dos três domínios do instrumento utilizado, pode-se notar médias inferiores àquelas apresentadas em estudo com esse público (Tomaschewski-Barlem et al., 2014)

Quanto ao estilo de vida desses estudantes, notou-se que uma parcela dos estudantes analisados apresentou baixos índices de adesão à prática de atividade física. Esse resultado representa uma tendência de sedentarismo mundial na população (Guerra, Farias Júnior, e Florindo, 2016). 
Outro ponto é que existe correlação na literatura entre a prevenção de $\mathrm{SB}$ e a realização de atividades físicas (Freitas, Carneseca, Paiva, e Paiva, 2014).

Neste estudo, na análise do estilo de vida dos estudantes, observou-se frequência alta para a alteração na nutrição. A nutrição é um elemento importante para elaboração da saúde do indivíduo como um todo, incluindo a saúde mental. Estudo com universitários de medicina identificou que a Síndrome de Burnout foi associada ao comportamento alimentar de risco (GalvánMolina et al., 2017).

Os resultados também demonstraram correlações negativas para as dimensões de exaustão emocional e descrença, e correlações positivas para eficácia profissional ao associar-se o estilo de vida desses estudantes à presença de $\mathrm{SB}$, concordando, dessa forma, com a literatura. Isso pode estar associado ao estilo de vida fantástico propor variáveis que seriam ideais a um sujeito que leva uma vida saudável. Assim sendo, um estilo de vida adequado seria protetor para desenvolvimento da SB (Fernández Sánchez, Pérez Mármol, \& Peralta Ramírez, 2017).

As correlações de maior importância negativa estão identificadas entre o domínio da SB exaustão emocional e o grupo de estilo de vida, sono, cinto de segurança, estresse e sexo seguro. Acredita-se que os pontos mais importantes dessa correlação seja o estresse e o sono. A literatura entra em consenso sobre a importância do estresse na gênese da SB e de seus domínios, logo, justifica-se a relação negativa entre as fases. Da mesma maneira, encontra-se o sono como importante fator protetor ao surgimento da Síndrome de Burnout e, portanto, também justificando a correlação negativa (GalvánMolina et al., 2017). Outra correlação observada foi a do domínio descrença da SB e da variável do estilo de vida cigarro e drogas. A relação fortemente negativa entre esses grupos pode ser explicada devido ao despertar da descrença em jovens ser geralmente acompanhada do início no mundo das drogas. Isso é geralmente causado porque estes podem acreditar que as drogas podem oferecer força para enfrentar um quadro emocional grave que passam ou até mesmo fuga. A Pesquisa Nacional de Saúde da Escola do Adolescente realizada com uma amostra nacional de 109.104 estudantes observou que as características da saúde mental foram associadas ao uso de tabaco, álcool e drogas ilícitas (Malta et al., 2014). Também foram identificadas relações fortemente positivas para eficácia profissional e família e amigos, sono, cinto de segurança, estresse e sexo seguro.
Essas relações contrariam a tendência das variáveis do estilo vida de se relacionarem negativamente com as variáveis da SB porque é a baixa eficácia profissional que determina a síndrome. Logo, esses fatores determinariam uma alta eficácia profissional. Isso se dá principalmente pelos elementos sono, proximidade com família e amigos e estresse, sendo esses fatores importantes para um bom desenvolvimento profissional (Fernández Sánchez et al., 2017; Galván-Molina et al., 2017).

As limitações encontradas neste estudo foram identificadas principalmente, na característica principal do estudo, que se trata de um estudo transversal, o que permite uma avaliação pouco profunda da condição estudada, enquanto uma abordagem longitudinal seria ideal para elencar as causalidades de melhor forma. Além disso, foram observadas limitações quanto à aplicação do instrumento de coleta de dados em grupo, que não permitiu maior privacidade ao jovem ao responder, podendo causar sensação desconfortável e, assim, interferir na lisura das respostas durante a avaliação.

Apesar de suas limitações, esta pesquisa foi positiva, uma vez que permitiu compreender a prevalência da SB em jovens e entender que fatores importantes acerca da vida cotidiana podem precipitar a síndrome e, por conseguinte, propiciar o abandono ou o baixo rendimento escolar.

O estudo buscou também contribuir com a produção científica acerca do tema, bem como sensibilizar outros para a realização de mais pesquisas, além de subsidiar políticas para o desenvolvimento de estratégias que venham não apenas aplacar a desordem orgânica, mas visem incentivar a prática de atividade física, incentivo à alimentação equilibrada e ressaltar a importância do sono, todos demonstrados como fatores protetores contra a SB.

\section{CONCLUSÃO}

Neste estudo foi identificada uma prevalência da Síndrome de Burnout na amostra de estudantes do ensino médio. Pode-se inferir que uma boa relação familiar, prática de atividade física e nutrição saudável, a negação do uso de álcool, drogas e cigarro, bem como a realização de práticas saudáveis de convivência na sociedade, são elementos de um estilo de vida determinado como positivo correlacionados diretamente com a Síndrome de Burnout e/ou algum dos seus sintomas. Assim, a estimulação dessas práticas deve ser realizada rotineiramente. 
Os resultados evidenciam a necessidade de ações de promoção de saúde e bem-estar dos estudantes com a participação de professores, profissionais de saúde e comunidade. A implementação de medidas que identifiquem precocemente sintomas de estresse e promovam controle das causas nessa população pode garantir a saúde mental no ambiente escolar, com impacto positivo na qualidade de vida e no desenvolvimento humano saudável.

\section{REFERÊNCIAS BIBLIOGRÁFICAS}

Chagas, M. K. S., Moreira Junior, D. B., Cunha, G. N., Caixeta, R. P., e Fonseca, E. F. (2016). Ocorrência da Síndrome de Burnout em acadêmicos de medicina de instituição de ensino no interior de Minas Gerais. Revista de Medicina e Saúde de Brasília, 5(2), 234-245. Disponível em: https://portalrevistas.ucb.br/index.php/ rmsbr/article/view/7241

Costa, E. F. O., Santos, S. A., Santos, A. T. R. A., Melo, E. V \& Andrade, T. M. (2012). Burnout Syndrome and associated factors among medical students: a crosssectional study. Clinics, 67(6), 573-580. Doi: 10.6061/ clinics/2012(06)05

Faria, R. R., Weber, L. N. D., e Ton, C. T. (2012). O estresse entre vestibulandos e suas relações com a família e a escolha profissional. Psicologia Argumento, 30(68), 43-52.Disponível em: https://periodicos.pucpr.br/index.php/psicologiaargumento/article/view/19893

Fernández Sánchez, J. C., Pérez Mármol, J. M., Peralta Ramírez, M. I. (2017). Influence of sociodemographic, occupational and life style factors on the levels of burnout in palliative care health professionals. Anales del Sistema Sanitario de Navarra, 40(3), 421-431. Doi: 10.23938/ASSN.0114.

Freitas, A., Carneseca, E., Paiva, C., e Paiva, B. (2014). Impacto de um programa de atividade física sobre a ansiedade, depressão, estresse ocupacional e síndrome de Burnout dos profissionais de enfermagem no trabalho. Revista Latino-Americana de Enfermagem, 22(2), 332-336. Disponível em: http://www.scielo.br/pdf/rlae/ v22n2/pt_0104-1169-rlae-22-02-00332.pdf
Galdino, M. J. Q., Martins, J. T., Haddad, M. C. F. L., Robazzi, M. L. C. C., e Birolim, M. M. (2016). Síndrome de Burnout entre mestrandos e doutorandos em enfermagem. Acta Paulista de Enfermagem, 29(1), 100-106. Disponível em: http://www.scielo.br/pdf/ape/ v29n1/1982-0194-ape-29-01-0100.pdf

Galván-Molina, J. F., Jiménez-Capdeville, M. E., Hernández-Mata, J. M., \& Arellano-Cano, J. R. Psychopathology screening in medical school students. (2017). Gaceta Médica de México, 153(1), 75-87. Disponível em: https://pdfs.semanticscholar.org/9287/a252eac617cf7dc28d5cea92da4a4a37a641.pdf

Gonzaga, L. R. V, Silva, A. M. B., e Enumo, S. R. F. (2016). Ansiedade de provas em estudantes do Ensino Médio. Psicologia Argumento, 34(84), 76-88. Doi: 10.7213/psicol.argum.34.084.AO07

Guerra, P. H., Farias Júnior, J. C., e Florindo, A. A. (2016). Comportamento sedentário em crianças e adolescentes brasileiros: revisão sistemática. Revista de Saúde Pública, 50(9), 1-15.

Magri, L. V, Melchior, M. O., Jarina, L., Simonaggio, F. F., e Bataglion, C. (2016). Relação entre sinais e sintomas de disfunção temporomandibular e de síndrome de Burnout em estudantes de odontologia. Revista Dor, 17(3), 171-177. Doi: 10.5935/1806-0013.20160065

Malta, D. C., Oliveira-Campos, M., Prado R. R., Andrade, S. S., Mello, F. C., Dias, A. J., e Bomtempo, D. B. (2014). Psychoactive substance use, family context and mental health among Brazilian adolescents, National Adolescent School-based Health Survey (PeNSE 2012). Revista Brasileira de Epidemiologia, 17(Suppl 1), 46-61. Doi: 10.1590/1809-4503201400050005

Maroco, J., e Tecedeiro, M. (2009). Inventário de Burnout de Maslach para Estudantes Portugueses. Psicologia, Saúde e Doenças, 10(2), 227-235. Disponível em: http://repositorio.ispa.pt/handle/10400.12/1090

Marques, C. P., Gasparotto, G. S., e Coelho, R. W. (2015). Líquen plano oral: reconhecendo a doença e suas características básicas. Salusvita, 34(1), 99-108. Disponível em: https://secure.usc.br/static/biblioteca/salusvita/ salusvita_v34_n1_2015_art_06.pdf 
Ovchinnikov, Y. V., Palchenkova, M. V., \& Kalachev, O. V. (2015). Burnout syndrome: diagnosis, principles of treatment, prophylaxis. Voenno-Meditsinskii Zhurnal, 336(7), 17-24. Disponível em: https://www.ncbi.nlm. nih.gov/pubmed/26821457

Rodriguez Añez, C. R., Reis, R. S., e Petroski, E. L. (2008). Versão brasileira do questionário "estilo de vida fantástico": tradução e validação para adultos jovens. Arquivos Brasileiros de Cardiologia, 91(2), 102-109. Doi: 10.1590/S0066-782X2008001400006

Tomaschewski-Barlem, J., Lunardi, V., Lunardi, G., Barlem, E., Silveira, R., e Vidal, D. (2014). Síndrome de Burnout entre estudantes de graduação em enfermagem de uma universidade pública. Revista LatinoAmericana de Enfermagem, 22(6), 934-941. Doi:: 10.1590/0104-1169.3254.2498
Tuominen-Soini, H., \& Salmela-Aro, K. (2014). Schoolwork engagement and burnout among Finnish high school students and young adults: profiles, progressions, and educational outcomes. Developmental Psychology, 50(3), 649-662. Doi: 10.1037/a0033898

\section{Fonte de Financiamento}

Recursos financeiros e bolsas de iniciação científica da Fundação de Amparo à Pesquisa do Estado de Minas Gerais (Fapemig).

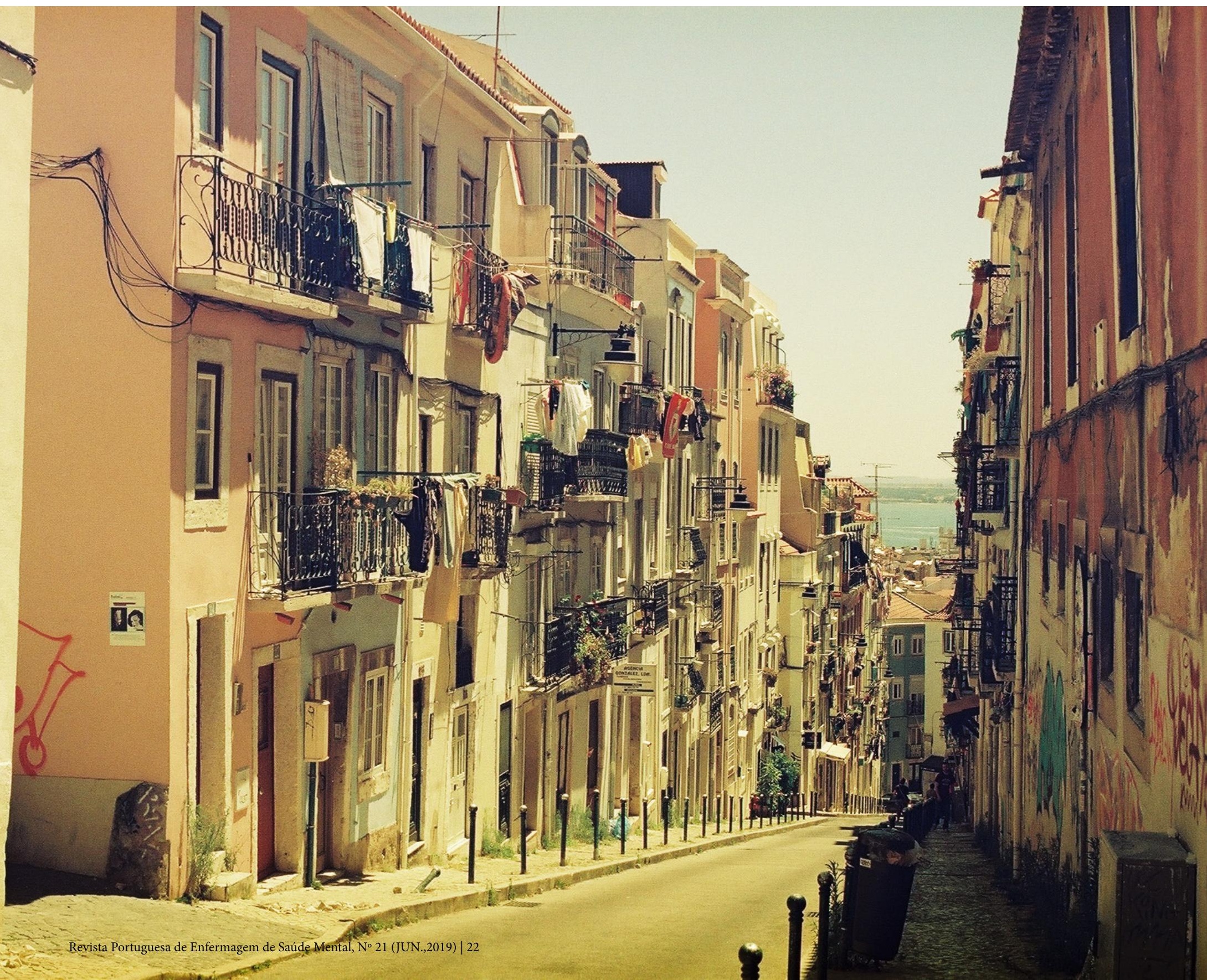

